\title{
REVISÃO DE MÉTODOS CONVENCIONAIS DE CONTROLE DE QUALIDADE BIOLÓGICO DE BIOFÁRMACOS DE ORIGEM RECOMBINANTE E BIOSSIMILARES E PERSPECTIVAS DE MÉTODOS ALTERNATIVOS
}

\author{
Marcos Luengo-Blanco' \\ 'Universidade Cruzeiro do Sul \\ Contato: marcos.luengo@cruzeirodosul.edu.br
}

Recebido em: abr. 2018; aceito jun. 2018; publicado out. 2018.

\section{Resumo}

A produção de biossimilares ou biofármacos com patentes expiradas pode ser uma oportunidade de crescimento para a indústria biofarmacêutica no Brasil. Entende-se por atividade biológica a habilidade ou capacidade específica do produto em obter um efeito biológico definido. Ela serve a múltiplos propósitos, na avaliação da qualidade do produto, sendo necessária para a caracterização e análise de medicamentos biossimilares, quando de sua comparação com um produto referência. Nessa breve revisão, o principal objetivo é explorar de forma mais critica o desenvolvimento e padronização de novas metodologias, que possam avaliar a atividade biológica de biofármacos e biossimilares, sob a luz de rever os principais modelos celulares utilizados, para desafiar a potencialidade dessas moléculas de atividade biológica, comparado com os modelos clássicos de células da linhagem murinas ou leveduras. A evolução de estudos bioanalíticos em torno desse assunto poderá contribuir para a disponibilização de testes de atividade biológica de maior precisão, gerando resultados mais conclusivos e seguros para os testes clínicos de fase I, II e III, no processo de regulamentação dos biossimilares. Além disso, futuramente a aplicação de testes de avaliação biológica com mais recursos analíticos, poderão ser indicados também como referência metodológica para classes diversas medicamentosas de biofármacos.

Palavras-chaves: Biofármacos. Biossimilares. Métodos alternativos.

\section{Biotecnologia e medicamentos biotecnológicos}

Biotecnologia pode ser definida como o uso dos organismos vivos para solucionar problemas ou desenvolver produtos novos, úteis, processos ou serviços ${ }^{[1]}$. Estes podem englobar tecnologias de diversos níveis, desde uma fermentação para a produção de alimentos e bebidas até tecnologias modernas de manipulação genética que resultem na produção de proteínas com aplicações terapêu- ticas ${ }^{[2]}$. A biotecnologia moderna surgiu no início dos anos 1970 como resultados de descobertas científicas no campo da engenharia genética e se tornou uma das maiores ferramentas de pesquisa científica. O desenvolvimento de inovação industrial no campo da biotecnologia moderna se apoia na compreensão de princípios científicos fundamentais, e sua base científica foi resultado do desenvolvimento da biologia molecular durante o século XX. Essa tecnologia revolucionou as ciências biológicas sobre tudo a capacidade de clonar 
genes humanas em microrganismos, embora esse impacto possa ser percebido em diversas áreas do conhecimento, uma de suas maiores influências foi sentida na área de medicamentos, surgindo a utilização do termo fármaco biotecnologia, para designar a aplicação da biotecnologia em ciências farmacêuticas ${ }^{[3]}$. Medicamentos biológiocos de origem recombinantes ou medicamentos biotecnológico são aqueles produzidos a partir de sistemas vivos, que possuem em formulação peptídeos, proteínas ou ácidos nucléicos (DNA e RNA) de ação farmacológica denominados biofármacos. Essas biomoléculas possuem estrutura molecular extensa, complexa e inerentemente heterogênea [4]. Os primeiros biofármacos surgiram na década de 1980 e vêm ocupando atualmente um espaço cada vez maior no mercado farmacêutico. O Food and Drug Administration (FDA), órgão de administração federal dos EUA, aprovou a insulina humana como o primeiro produto biológico para uso clínico em seres humanos, a qual simboliza um marco na história da revolução farmacobiológica, desenvolvida pela Genentech, mas licenciada pela Eli Lilly, que a produz desde 1982. Naquela época, o produto tinha um mercado de aproximadamente 700 milhões de dólares anuais somente nos EUA. No Brasil esse trabalho foi realizado pelo grupo do professor Spartaco Astolfi Filho, com financiamento da Biobrás, do Programa de Apoio ao Desenvolvimento Científico e Tecnológico e da FINEP, dentro do convênio UnB/Biobrás e patenteado nos EUA ${ }^{[5]}$.

Outro exemplo é o caso da Eritropoietina, empregada no tratamento de anemia e coadjuvante no tratamento do Câncer. Atualmente essa substância, que obteve registro em 1989, é o biofármaco mais vendido no mundo, fatura uma receita anual superior a US\$ 10 bilhões. Segundo os biofármacos mais importantes são: Fatores sanguíneos: (Fator VIII e IX) e Agentes tromboembolíticos, Hormônios: (Insulina, Hormônio de Crescimento, Gonadotróficos), Citocinas: (Interferon- $\alpha, \beta$, $\gamma$, Interleucinas); Fatores de crescimento hematopoiéticos: (Eritropoetina, Fatores estimuladores de colônias hematopoiéticas); Fatores de crescimento: (Fator de crescimento epidérmico); Imu- nobiológicos: (Soros, Vacinas e Anticorpos monoclonais) e Enzimas terapêuticas: (Asparaginase, DNase, Superóxido dismutase, Lactase). Atualmente, cinco entre os dez medicamentos mais vendidos no mundo já são biológicos e as vendas de medicamentos produzidos por biotecnologia representam $31 \%$ das vendas dos 100 medicamentos mais vendidos. Esta proporção deve subir para $40 \%$ em 2018, com oito produtos biotecnológicos, dos quais seis anticorpos monoclonais, entre os dez mais vendidos, segundo o boletim da ANVISA em 2017. Os biofármacos atualmente aprovados para comercialização são utilizados para o tratamento ou prevenção de doenças como mal de Alzheimer, apneia do sono, artrite reumatoide, ataques cardíacos, Câncer de mama, câncer renal, dermatite atópica, diabetes, doença de Crohn, esclerose múltipla, fibrose cística, hemofilia, hepatite, enfarte cerebral ou apoplexia, insuficiência cardíaca, hanseníase, leucemia, leucemia linfocítica crônica, linfomas, lúpus e tumores cerebrais ${ }^{[6]}$.

\section{Medicamentos biossimilares}

Medicamentos biológicos chamados de biossimilares ou também "Follow-On Protein Product" na Europa é o termo usado para se referir ao biofármaco não inovador, portanto, os biossimilares são cópias de um biofármaco que tiveram sua patente expirada, e que demonstra ter uma eficácia similar e o mesmo perfil de segurança em humanos. O termo medicamento "genérico" é usado para descrever produtos medicamentosos químicos (produtos sintéticos) de pequenas moléculas que são estrutural e terapeuticamente equivalentes a um produto de referência cuja patente e/ou período de proteção de dados tenha expirado. A demonstração de bioequivalência do medicamento genérico com um produto de referência é geralmente adequada e suficiente para inferir a equivalência terapêutica entre o medicamento genérico $e$ o produto de referência. No entanto, a abordagem estabelecida para os medicamentos genéricos não é adequada para o desenvolvimento, avaliação e licenciamento dos medicamentos biossimilares, uma vez que os biotecnológicos consistem de pro- 
teínas complexas e relativamente grandes que são difíceis de caracterizar ${ }^{[78]}$.

O desempenho clínico dos medicamentos biológicos é muito influenciado pelo processo de fabricação e alguns estudos clínicos também são necessários para sustentar a segurança e a eficácia de um medicamento biossimilar. Como acontece com qualquer programa de desenvolvimento de medicamento, o desenvolvimento de um medicamento biossimilar, envolve uma abordagem gradual que começa com a caracterização e avaliação dos atributos de qualidade do produto, seguido por estudos pré-clínicos e clínicos ${ }^{[9]}$. Os padrões estabelecidos pelo Comitê de Especialistas para a Padronização Biológica (CEPB) da Organização Mundial da Saúde servem como base para o estabelecimento dos requisitos nacionais para a produção, controle de qualidade e regulação em geral dos medicamentos biológicos. Além disso, os Padrões Internacionais de medida são ferramentas essenciais para o estabelecimento da potência dos medicamentos biológicos em todo o mundo ${ }^{[10,11]}$. Para testes de potência ou atividade biológica de um medicamento biossimilar, deve ser avaliado a partir de estudos comparativos entre um biofármaco de referência, frente ao produto biológico similar a fim de demonstrar a similaridade em termos de qualidade, segurança e eficácia. O produto biológico de referência deve fornecer a base para o estabelecimento dos perfis de segurança, qualidade e eficácia ao qual o produto biológico similar é comparado. Assim, também se estabelece, a seleção da dose e via de administração, onde esses dados são utilizados para sustentar a solicitação de licenciamento do medicamento similar em teste [12,13].

O delineamento dos estudos de eficácia e segurança, são demonstrados em ensaio(s) clínico(s) devidamente controlado(s), randomizado(s) e com poder estatístico adequado. Os estudos clínicos devem ser preferencialmente do tipo duplo-cego, ou, no mínimo, observador-cego. Na ausência de qualquer fator cego, uma justificativa cuidadosa será necessária para provar que os resultados do estudo estão livres de viés significativo. Em princípio, os desenhos de equivalência (que requerem limites inferior e superior de comparabilidade) são claramente os preferidos para a comparação de eficácia e segurança do biossimilar comparado ao medicamento referência. Já os aspectos relacionados à qualidade do produto, a diretriz da OMS é clara ao definir que a comparação da qualidade mostrando similaridade molecular entre o produto biológico similar e o produto biológico de referência é indispensável para fornecer justificativas para a previsão do perfil de segurança e eficácia clínica; Pequenas diferenças no processo de fabricação podem afetar a farmacocinética, farmacodinâmica, eficácia e/ou segurança dos produtos biológicos.

Outro aspecto bastante relevante das características de biofármacos, refere-se ao potencial imunogênico dos produtos biotecnológicos. A resposta imune contra um produto biológico é influenciada por muitos fatores, tais como a natureza do princípio ativo (principalmente os proteináceos), impurezas relacionadas ao produto e ao processo, excipientes e estabilidade do produto, via de administração, regime de dose, e fatores relacionados ao paciente, à doença e à terapia. As consequências da imunogenicidade indesejada podem variar consideravelmente, desde clinicamente irrelevante até grave e potencialmente fatal. Portanto, a imunogenicidade dos produtos biológicos deve sempre ser investigada antes do licenciamento. Mesmo que a eficácia e a segurança entre um biossimilar e um produto referência tenham se mostrado semelhantes, a imunotoxicidade pode ainda ser diferente ${ }^{[14]}$.

\section{Controle de qualidade biológica de produtos biotecnológicos}

As metodologias aplicadas em controle de qualidade de biofármacos, segue o mesmo perfil de ensaios de um medicamento comum, a maioria dos ensaios de caráter físico-químicos, são realizados utilizando metodologias instrumentais para avaliação quantitativa e qualitativa da natureza proteica de biomoléculas complexas. Para o controle microbiológico são pesquisados praticamente os mesmos parâmetros microbiológicos realizados 
em medicamentos que exigem esterilidade ou de não estéreis, com a exceção pesquisa de possíveis contaminações por toxinas microbianas de origem do sistema biológico de produção ${ }^{[15]}$. Uma vez que os produtos biotecnológicos de origem recombinante podem possuir variabilidades biológicas características, se faz necessário e obrigatório a realização de ensaios biológicos para garantir a qualidade biológica do processo de desenvolvimento e registro de novo produto biológico referencial ou biossimilar, assim como na rotina de produção, com o objetivo de detectar alterações em sítios específicos da macromolécula que podem repercutir na ineficiência do medicamento. Além de avaliar a ação farmacodinâmica do medicamento biológico, demonstrado em bioensaios baseado em cultivos celulares capazes de detectar a interação das moléculas terapêuticas e receptores específicos através de modelos celulares, possibilitando a medida de potência, assim como avaliar as características biológicas da evolução da célula tratada, tendo em conta os desvios estatísticos. Já para produtos com múltiplas atividades biológicas, esse produto deve atender um conjunto de ensaios funcionais relevantes, que possam atestar sua eficácia, em suas diferentes ações farmacológicas, de acordo, com seu uso clínico ${ }^{[16]}$. De forma geral o estudo de qualidade de um medicamento biológico nao se restringe somente à atividade biológica, mas sim uma gama de outras metodologias analíticas específicas que devem ser aplicadas no controle de qualidade de biofármacos; Além disso, o ensaio da atividade biológica colabora suplementando os resultados das propriedades físico-químicas devido ao potencial e adequação do método utilizado para confirmar a complexidade estrutural da proteína, (mostrando relação estrutura atividade ${ }^{[16]}$. No entanto, a natureza dos ensaios biológicos, podem possuir uma elevada mutabilidade, devido aos agentes vivos utilizados para essas determinações analíticas, que devem ser levadas em consideração de forma rigorosa, principalmente o controle de bancos celulares utilizados para nas metodologias bioanalíticas. Os resultados dos ensaios biológicos deverão ser fornecidos e expresso em unidades de atividade. Calibrados com valores de padrões de referência internacional ou nacional, de acordo, com suas especificações de fabricação ${ }^{[17]}$. E por fim obrigatoriamente os estudos clínicos de fase I, II e III são preconizados tanto para o registro do produto referência como para o produto biossimilar, com o objetivo de apontar as diretrizes para estabelecer critérios de eficácia e segurança desses medicamentos, principalmente frente aos possíveis efeitos tóxicos in vivo humano. Portanto, pode ser considerado que um medicamento biológico biossimilar quando a comparado, com o produto farmacêutico de referência, por métodos adequados apresentando exatidão e precisão, mostram diferenças funcionais significativas, promovendo maior segurança da qualidade ao medicamento avaliado ${ }^{[18]}$.

\section{Propostas de métodos alternativos para avaliação biológica de produtos biotecnológicos e seus biossimilares}

De acordo com Agência Nacional de Vigilância Sanitária (ANVISA), os medicamentos biológicos não são completamente caracterizados por análises químicas convencionais. "São necessários ensaios bioquímicos, imunológicos e ensaios biológicos in vitro e in vivo para avaliar sua atividade". Embora a qualidade dos métodos analíticos para caracterização de proteínas tenha aumentado drasticamente ao longo das últimas décadas, ainda existem obstáculos à completa caracterização de produtos bioterápicos complexos. Esses dados mostram a importância de testes de qualidade biológica mais detalhados e aprofundados, que podem estabelecer padrões de homogeneidade referente à ação biológica desses produtos frente às suas etapas de produção ${ }^{[19]}$. Diante disso, através de aplicações biotecnológicas podem ser elaboradas, metodologias que possam ampliar os conhecimentos científicos em controle de qualidade biológico no Brasil, pois a capacitação da mão de obra constitui outro desafio na indústria brasileira. Outro ponto significativo tanto para o desenvolvimento e validação de métodos bioanalíticos, são os modelos celulares utilizados para a realização de metodologias que envolvem sistemas cultivo celular, para a avalia- 


\section{Brazilian Journal of Natural Sciences | Versão On-line ISSN 2595 - 0584 \\ Edição no 1- vol. 3 - outubro 2018 | www.bjns.com.br}

ção de atividade biológica, as quais são sempre recomendados por várias farmacopeias como por exemplo: americana, europeia, japonesa e chinesa $[20,21,22]$. No entanto, $\mathrm{o}$ índice de discordância entre os resultados de atividade biológica in vitro e in vivo utilizando as linhagens celulares oficialmente recomendas, são bastante significativos. Nesse sentido, existe atualmente uma real necessidade de adaptação e refinamento, dessas metodologias indicadas pelas principais farmacopeias, explorando novos modelos celulares com características fenotípicas mais humanas, já que os principais modelos celulares hoje utilizados são de origem murina. Além disso, a aplicação de outras metodologias complementares que possam avaliar o grau de comprometimento, diferenciação e transdução de sinais promovidos pela ação dos produtos biológicos ou biossimilares, através de métodos moleculares mais sensíveis e precisos, permitirão inovações no setor de controle de qualidade de medicamentos, através da implantação e validação de métodos que utilizam biologia molecular analítica, tecnologia de cultura celular e citometria de fluxo. Nesse contexto, essa proposta atende às questões éticas sobre a experimentação animal, implantando ensaios in vitro de caráter pré-clínico, que possuem especificidade, produtividade da técnica e menor custo, por serem in vitro dispensando o uso de animais, praticando o conceito dos "3Rs" ("replacement, reduction e refinement"), isto é, substituição, redução e refinamento, no que se refere aos animais utilizados em experimentos, preconizando técnicas humanitárias, considerado como uma referência para a ciência contemporânea, de acordo com a Rede Nacional de Métodos Alternativos (RENAMA). Outro aspecto positivo é que esse estudo atende às questões éticas sobre a experimentação animal, implantando ensaios que possuem especificidade, produtividade da técnica e menor custo por serem in vitro, dispensando o uso de animais, preconizando técnicas humanitárias, consideradas como referência para a ciência contemporânea ${ }^{[23]}$.

Por fim, a elaboração e validação de metodologias empregadas na avaliação biológica, são de fundamental importância e colaboram com as
Boas Práticas de Fabricação dos produtos biossimilares no nosso país e a capacitação da mão de obra constituem um grande desafio na indústria brasileira. Além disso, os biossimilares podem ser peça chave para a saúde pública, disponibilizando o mesmo tratamento farmacoterapêutico, com a mesma qualidade do medicamento referencial e com menor custo. Estudos na literatura mostram que biossimilares, pré-aprovados em testes préclínicos não correspondem à mesma performance em testes clínicos humanos, resultando na exclusão dos processos de aprovação para o registro. A não coerência entre estudos in vivo e in vitro, segundo o "European regulatory guidelines for biosimilars", sugere possíveis falhas nos instrumentos de avaliação que geralmente se dão pela falta de sensibilidade dos métodos in vitro utilizados ${ }^{[25]}$.

\section{Conclusão}

Diante de todo o exposto, pode-se concluir que apesar dos grandes avanços biotecnológicos no âmbito farmacêutico, além de aumentar a produção de insumos, e disponibilização de novos produtos, no entanto o grande desafio ainda persiste na expansão de desenvolvimento e padronização de métodos bioanalíticos, que possam garantir a qualidade desses novos medicamentos. Portanto, se faz necessário uma revisão crítica de estudos comparativos entre os modelos celulares murinos utilizados para a realização da atividade biológica de biofármacos, com outras fontes celulares alternativas que possuem características fenotípicas mais próximas de humanos, aproximando mais os métodos in vitro ao cenário fisiológico humano. Os estudos de controle de qualidade de um medicamento, devem acompanhar as novas tendências tecnológicas de produção, como é caso dos medicamentos biológicos, os quais possuem perfis e mecanismos de ação mais complexos e suscetíveis a diversos tipos de alterações comprometendo sua eficácia e segurança. Futuramente esses estudos não só disponibilizarão ferramentas analíticas de grande precisão em testes de atividade biológica, como também poderão ser usados para avaliação de efeitos adversos ou como recursos analíticos 
para identificação de novos biomarcadores farmacológicos. E por fim, o mercado biotecnológico farmacêutico brasileiro vem aumentando gradativamente, mas esse fato não garantiu a autonomia no desenvolvimento tecnológico, a elaboração e padronização de novos métodos bioanalíticos poderão contribuir para o avanço e desenvolvimento na área de biossimilares nacionais, que possuem elevado impacto nas áreas da saúde e econômica brasileira, bem como o contexto do desenvolvimento, domínio científico e independência tecnológica do país.

\section{Referências Bibliográficas}

1. Chooi SS, Katsuyama Y, Bai L, Deng Z, Ohnishi T, Kim ES. Genome engineering for microbial natural product discovery. Curr. Opin. Microbiol. 2018;(45):53-60.

2. BORZANI, W. et al. Biotecnologia Industrial: Engenharia Bioquímica. 1 ed. São Paulo: Edgard Blucher Ltda, 2001. v. 2.

3. Korte C, Quadbach J. Formulation development and process analysis of drug-loaded filaments manufactured via hot-melt extrusion for 3D-printing of medicines. Pharm Dev Technol. 2018;(9):1-11.

4. Parr MK, Montacir O, Montacir H. Physicochemical characterization of biopharmaceuticals. J Pharm Biomed Anal. 2016;(130):366-389.

5. Torres-Freire C, Golgher D, Callil V. Biotecnologia em saúde humana no Brasil: produção científica e pesquisa e desenvolvimento. Dossiê desenvolvimento e inovação. Novos estud. 2014; CEBRAP n ${ }^{\circ} .98$ São Paulo Mar.

6. Rantanen, J, Khinast J. 2015. The Future of Pharmaceutical Manufacturing Sciences. J. Pharm. Sci., 2014; (11), 3612-3638.

7. Savla R, Browne J, Plassat V, Wasan KM, Wasan EK. Review and analysis of FDA approved drugs using lipid-based formulations. Drug Dev Ind Pharm. 2017; (11):1743-1758.

8. Brasil. Agência Nacional de Vigilância Sanitária. Resolução da Diretoria Colegiada (RDC) no135 de 18 de maio de 2011.

9. Grabowski HG, DiMasi JA, Long G. The roles of patents and research and development incentives in biopharmaceutical innovation. Health Aff (Millwood). 2015;(2):302-10.

10. Elder DP, Kuentz M, Holm R. Pharmaceutical excipients - quality, regulatory and biopharmaceutical considerations. Eur J Pharm Sci. 2016;(87):88-99.

11. World Health Organization WHO Expert Committee on Biological Standardization. 2012;(964):1-228.

12. Bridges SL Jr., White DW, Worthing $A B$, Gravallese EM, O’Dell JR, Nola K, Kay J, Cohen SB. The Science Behind Biosimilars: Entering a New Era of Biologic Therapy. Arthritis Rheumatol. 2018;(3):334-344.

13. Brasil. Agência Nacional de Vigilância Sanitária. Resolução de Diretoria Colegiada (RE) no 899, de 29 de maio de 2003.

14. Turecek PL, Bossard MJ, Schoetens F, Ivens IA. PEGylation of Biopharmaceuticals: A Review of Chemistry and Nonclinical Safety Information of Approved Drugs. J Pharm Sci. 2016;(2):460-475.

15. Pinto TJA, Kaneko TM, Pinto AFT. Controle biológico de qualidade de produtos farmacêuticos, correlatos e cosméticos. São Paulo, Ateneu, 2015, $7805 \mathrm{p}$.

16. Brinks V, Hawe A, Basmeleh AH, et al. Quality of original and biosimilar epoetin products. Pharm Res. 2011;28(2):386-93.

17. Walsh G. Biopharmaceuticals. Biochemistry and Biotechology. 2 ed. Chichester: Wiley, 2003. $551 \mathrm{p}$.

18. Zuñiga $L$, Calvo B. Biosimilars approval process. Regul Toxicol Pharmacol. 2010;(3):374-7.

19. Arakawa H. Development of Highly Sensitive Analytical Methods for Biologically Relevant Materials and Their Pharmaceutical Applications. Chem Pharm Bull. 2017;12):1099 1112.

20. European pharmacopeia. London: EDQM. 2012; 7 th, 2015-2018.

21. British pharmacopeia. London: HMSO. 2011; v. I e II. (Ph. Eur. Monograph 2206).

22. Chinese Pharmacopoeia English Edition. 2010; 3: 266-269.

23. Cheluvappa R, Scowen P, Eri R. Ethics of 
animal research in human disease remediation, its institutional teaching; and alternatives to animal experimentation. Pharmacol Res Perspect. 2017;5(4).

24. Grabowski HG, DiMasi JA, Long G.The roles of patents and research and development incentives in biopharmaceutical innovation. Health Aff (Millwood). 2015; (2):302-310.

25. Cai XY, Gouty D, Baughman S, Ramakrishnan $M$, et al. Recommendations and requirements for the design of bioanalytical testing used in comparability studies for biosimilar drug development. Bioanalysis. 2011;3(5):535-40. 